\title{
Costos pegajosos (sticky costs) en empresas españolas: un estudio empírico
}

Eliana Werbin

Universidad Nacional de Córdoba, Facultad de Ciencias

Económicas, Instituto de Contabilidad emwerbin@yahoo.com.ar

\section{Luz María}

Marín Vinuesa

Universidad de la Rioja,

Departamento Economía y

Empresa

luz-maria.marin@unirioja.es

\section{Marcela Porporato}

York University School of Administrative Studies porpomar@yorku.ca

\section{Resumen}

Entender cómo y por qué cambia el nivel de costos es fundamental para administrar eficientemente una organización. Tradicionalmente, los costos se dividen en fijos y variables, según varíen o no con respecto al nivel de actividad. Sin embargo, los estudios empíricos más recientes muestran evidencia de costos "pegajosos" (sticky costs) porque su respuesta es más fuerte cuando el nivel de actividad incrementa que cuando dicho nivel disminuye. Este artículo comprueba que los costos pegajosos se observan en empresas españolas en el periodo 2005-2007 y que su magnitud es comparable a la documentada en estudios anteriores. Este mismo estudio puede ser replicado en otros contextos internacionales siempre y cuando se disponga de bases de datos completas y públicamente disponibles, lo que representa una interesante línea de investigación.

Palabras clave: costos pegajosos, contabilidad de costos, contabilidad administrativa, empresas españolas. 


\title{
An empirical study of sticky costs in Spanish companies
}

\begin{abstract}
To understand how and why the level cost changes is essential to efficiently manage an organization. Traditional cost accounting classifies them into fixed and variable, whether they change or not with changes in the activity level. Nevertheless, recent empirical research shows evidence of sticky costs because their response is stronger when the activity level increases that when such level falls. This paper shows that sticky costs are present in Spanish companies for the period 2005-2007 and that their magnitude is comparable with previous studies. This same type of study can be replicated in other international contexts provided that there are complete and publicly available databases, which means an interesting line of research.
\end{abstract}

Keywords: sticky costs, cost accounting, managerial accounting, Spanish companies.

\section{Costos pegajosos: ¿un nuevo concepto?}

Comprender el comportamiento de los costos es imprescindible para gestionar una empresa. La contabilidad tradicional de costos los divide en fijos y variables, según su respuesta ante variaciones en el nivel de actividad. Tradicionalmente ha venido admitiéndose que la relación entre costos variables y el nivel de actividad es simétrica (Hongren, 2002); es decir, se mantiene proporcional para todo tipo de variaciones en el factor, tanto para aumentos como para disminuciones en el mismo. Sin embargo, la evidencia empírica de los últimos años muestra que esto no es así, sino que los costos siguen un comportamiento "pegajoso" (sticky), ya que la respuesta de los costos ante incrementos en el nivel de actividad es más fuerte que la respuesta de los mismos ante las disminuciones.

A pesar de que el tema se percibe como nuevo, Malcom (1991) ya introdujo el concepto de costos pegajosos, indicando que

[...] muchos de estos costos nuevos tienden a ser [...] no estrictamente proporcionales con el cambio en la actividad. Un ejemplo muy común son los costos de orden y manejo de materiales. Mientras la producción crece se agregan empleados adicionales para manejar las tareas adicionales; pero, si la producción decrece, este personal no es inmediatamente despedido [...] Estos costos han sido rotulados algunas veces como costos pegajosos. 
Otro de los estudios que mencionan este concepto en las primeras épocas es el de Mak y Roush (1994) al considerar cómo el ABC (Costeo Basado en Actividades) afecta a los presupuestos flexibles. Remiten al estudio anterior de Malcom (1991); señalan que los costos pegajosos son los que pueden incrementarse en el corto plazo, pero no decrecen cuando declina la actividad.

Una de las primeras referencias empíricas a la idea de "costos pegajosos" puede encontrarse en Noreen y Soderstrom (1997), quienes probaron la exactitud predictiva del modelo de costos tradicional; encontraron que algunos costos tienen una respuesta menor ante una baja del volumen que ante un alza. Pero es a partir del trabajo de Anderson, Banker y Janakiraman (2003) que el fenómeno de los costos pegajosos ha despertado un renovado interés. Los estudios empíricos han comenzado a publicarse en estos últimos años, tal como se detalla en el cuadro 1 . Cabe destacar que hasta la fecha ningún estudio ha analizado la teoría de los costos pegajosos en España; además, hay una gran escasez de trabajos publicados o presentados en congresos referidos a costos pegajosos en idioma español, de ahí la contribución del presente artículo a la literatura existente.

Estos estudios muestran que la relación simétrica que la teoría asigna a los costos variables no es tal, pues estos costos son rígidos a la baja. Se sugiere que la magnitud del cambio en los costos no depende sólo de la magnitud del cambio en el factor generador del costo, sino también de la dirección en la que se produce dicho cambio (ascendente o descendente). Para definir esta situación, numerosos autores hablan de sticky costs (cuya traducción al español sería costos "pegajosos", según Werbin, 2009), indicando que un costo es pegajoso si la magnitud del incremento asociada con un aumento en el volumen de actividad es mayor que la magnitud de la caída asociada con una disminución del volumen. Esta relación de "costos pegajosos" se observa en empresas de diversos sectores, tanto en aquellas que operan en países desarrollados con estabilidad económica como en las que operan en entornos más turbulentos de economías emergentes.

\section{Comportamiento de los costos pegajosos}

La práctica empresarial reflejada en los estudios del cuadro 1 sugiere que los gerentes ajustan deliberadamente los recursos a los cambios que se producen en el volumen de actividad, medido éste según el nivel de ventas. De esta forma, podemos distinguir entre los costos que se mueven automáticamente con el volumen 


\section{Cuadro 1}

\section{Comportamiento de costos según la teoría y lo documentado empíricamente}

\begin{tabular}{|c|c|c|c|c|}
\hline Panel A & $\begin{array}{l}\text { Ámbito } \\
\text { de estudio }\end{array}$ & Modelo & $\begin{array}{l}\text { Efecto ante un } \\
\text { aumento en las ventas }\end{array}$ & $\begin{array}{c}\text { Efecto ante una } \\
\text { disminución en las } \\
\text { ventas }\end{array}$ \\
\hline Teoría & $\begin{array}{c}\text { Todo } \\
\text { tipo de } \\
\text { empresas }\end{array}$ & $\begin{array}{c}\text { Costos totales } \\
=\text { Costos fijos }+ \\
\text { Costos variables } \\
\text { unitarios } \mathrm{x} \\
\text { unidades vendidas }\end{array}$ & $\begin{array}{c}\text { Costos variables } \\
\text { aumentan un } \\
\text { determinado porcentaje } \\
\text { por cada } 1 \% \text { de } \\
\text { aumento en el nivel de } \\
\text { ventas }\end{array}$ & $\begin{array}{l}\text { Costos variables } \\
\text { disminuyen en el } \\
\text { mismo porcentaje } \\
\text { cuando las ventas } \\
\text { caen en un } 1 \%\end{array}$ \\
\hline $\begin{array}{l}\text { Panel B } \\
\text { Estudios } \\
\text { empíricos }\end{array}$ & $\begin{array}{c}\text { Ámbito } \\
\text { de estudio }\end{array}$ & Modelo & $\begin{array}{c}\text { Efecto ante un } \\
\text { aumento } \\
\text { en las ventas }\end{array}$ & $\begin{array}{c}\text { Efecto ante una } \\
\text { disminución en las } \\
\text { ventas }\end{array}$ \\
\hline $\begin{array}{c}\text { Anderson, } \\
\text { Banker y } \\
\text { Janakiraman } \\
(2003) \\
\end{array}$ & EE.UU. & $\begin{array}{c}\text { Comercialización } \\
\text { administración y } \\
\text { generales }\end{array}$ & Aumentan $0.55 \%$ & Disminuyen $0.35 \%$ \\
\hline $\begin{array}{c}\text { Ribeiro de } \\
\text { Medeiros y de } \\
\text { Souza Costa } \\
(2004)\end{array}$ & Brasil & $\begin{array}{c}\text { Comercialización } \\
\text { administración y } \\
\text { generales }\end{array}$ & Aumentan $0.59 \%$ & Disminuyen $0.32 \%$ \\
\hline $\begin{array}{c}\text { Calleja, } \\
\text { Steliaros y } \\
\text { Thomas (2006) }\end{array}$ & $\begin{array}{l}\text { EEUU, } \\
\text { Inglaterra, } \\
\text { Francia y } \\
\text { Alemania }\end{array}$ & Operativos & Aumentan $0.97 \%$ & Disminuyen $0.91 \%$ \\
\hline $\begin{array}{c}\text { Porporato y } \\
\text { Werbin (2010) }\end{array}$ & $\begin{array}{l}\text { Argentina, } \\
\text { Brasil y } \\
\text { Canadá } \\
\text { (Bancos) }\end{array}$ & Totales & $\begin{array}{c}\text { Aumentan: } 0.60 \% \\
\text { Arg., } 0.82 \% \text { Brasil y } \\
\text { 0.94\% Canadá }\end{array}$ & $\begin{array}{c}\text { Disminuyen: } 0.38 \% \\
\text { Arg., } 0.48 \% \text { Brasil y } \\
\text { 0.55\% Canadá }\end{array}$ \\
\hline $\begin{array}{l}\text { Banker, } \\
\text { Byzalov } \\
\text { y Plehn- } \\
\text { Dujowich } \\
(2010)\end{array}$ & 19 países & Operativos & Aumentan $0.87 \%$ & Disminuyen $0.76 \%$ \\
\hline
\end{tabular}


de actividad y aquellos otros que dependen de las decisiones de los gerentes. El principal fundamento de la existencia de los costos pegajosos es que ante situaciones de incertidumbre sobre la demanda futura de los productos de la empresa es probable que los gerentes decidan demorar la reducción de los costos hasta estar más seguros de la caída en el volumen de actividad. Ante una disminución en la actividad, los gerentes deben decidir entre mantener los recursos comprometidos y soportar los costos de operar con capacidad ociosa, o reducir los recursos comprometidos y soportar los costos de salida y de posterior reemplazo si la actividad se recupera. Esto sugiere que los costos serán más pegajosos cuanto menor sea la probabilidad de una declinación permanente del nivel de actividad y/o cuanto más oneroso sea el ajuste (salida y posterior reemplazo).

En la realidad empresarial los recursos no son perfectamente divisibles, no pueden agregarse o detraerse en pequeñísimas cantidades como para amoldarse exactamente al nivel de actividad. Sin embargo, este hecho por sí solo no ocasiona que los costos sean pegajosos. La existencia de costos pegajosos se debe a fuerzas opuestas en el ajuste de los recursos debido a la disminución en el nivel de actividad que los gerentes deciden no acompañar con una reducción de costos en términos proporcionales. También se sugiere que los costos pegajosos surgen porque hay una decisión de la gerencia de mantener recursos ociosos en el intervalo que va desde la reducción del volumen de actividad hasta el momento del ajuste. Asimismo, existe un lapso entre la decisión de reducir costos y el momento en que la reducción puede hacerse efectiva, por ejemplo, por cuestiones contractuales.

En los últimos años el tema de costos pegajosos ha sido investigado desde diversos ángulos. Banker, Ciftci y Mashruwala (2008) analizan cómo el optimismo (o pesimismo) en la dirección afecta a las decisiones de asignación de recursos, y sus resultados refuerzan el argumento de que los administradores deliberadamente ajustan estos recursos en respuesta a las condiciones observadas de la demanda. Otras investigaciones recientes han documentado que factores tales como la capacidad utilizada (Balakrishnan, Petersen y Soderstrom, 2004), la importancia del costo (Balakrishnan y Gruca, 2008), y los incentivos de construcción de un imperio (Chen, Lu y Sougiannis, 2007), moderan la respuesta asimétrica de los costos ante cambios en el nivel de actividad. También Wiersma (2010) encuentra que los costos son más pegajosos mientras más orientados al corto plazo están los incentivos/bonos de los gerentes. 
Hay otros estudios empíricos que se han enfocado en diferentes industrias, particularmente en la de salud. West (2003) investigó acerca de la presencia de costos pegajosos en el sector salud, tratando de identificar estrategias para manejarlos, concluyendo que los costos son más pegajosos cuando los administradores tienen menos control sobre ellos. Balakrishnan, Petersen y Soderstrom (2004) estudiaron la relación entre la magnitud del cambio y la capacidad utilizada en 49 centros de salud. Balakrishnan y Gruca (2008) examinaron el comportamiento de los costos para hospitales de Ontario, mostrando que los costos son más pegajosos si están más vinculados a servicios centrales.

Anderson, Banker y Janakiraman (2003) sugieren que cuando se estudia un periodo más amplio el efecto de los costos pegajosos es menos notorio. En periodos de análisis más amplios, los gerentes pueden tener mayor seguridad sobre la caída en el volumen de actividad, por lo que los costos de ajuste serán relativamente más pequeños que los costos de mantener recursos no utilizados. Cuanto más seguros estén los gerentes de que la caída en la actividad puede persistir durante varios periodos, menos pegajosos serán los costos; por el contrario, si los gerentes tienen la convicción de que la caída en la actividad es pasajera se mostrarán más reticentes a reducir costos y, por tanto, más pegajosos serán éstos. Asimismo, se ha argumentado que el optimismo (o pesimismo) de los gerentes puede afectar a sus decisiones sobre localización de recursos.

Por otra parte, también se ha planteado que cuanto más intensivo es el uso del capital y la mano de obra, más pegajosos tienden a ser los costos debido a que el ajuste (venta de activos, cancelación de contratos a largo plazo, despido de empleados) es más oneroso. En este sentido, hay costos más y menos pegajosos, dependiendo de la facilidad que pueda tener la empresa para deshacerse de ellos ante una caída en la actividad. Esto último depende no sólo de la estructura de costos de cada empresa, sino que en la mayoría de los casos depende del sector de la economía o del tipo de actividad desarrollada. También se ha documentado la relación entre la función de los costos y su comportamiento, de manera que cuanto más vinculada está dicha función con el negocio central (core business) más difícil es reducir sus costos (Balakrishnan y Gruca, 2008).

El modelo de costos pegajosos también ha sido cuestionado desde bases empíricas y teóricas. Con respecto a los estudios empíricos, pueden mencionarse a Anderson y Lanen (2007), quienes junto a Balakrishnan y Soderstrom (2010) y Weiss (2010) no encuentran evidencia de costos pegajosos y cuestionan el vínculo entre costos 
pegajosos y el comportamiento de los administradores. Por otra parte, la idea de que los costos pegajosos se deben a acciones deliberadas de la dirección también ha sido recientemente cuestionada desde una perspectiva teórica por Anderson y Lanen (2007), argumentando que el modelo mecánico de cambios en los costos no es una teoría porque todos los ajustes de costos son el resultado de decisiones pasadas de la administración. Más allá de esto, la evidencia empírica existente en costos pegajosos parece ser robusta para muestras agregadas, aunque menos consistentes en la medida en que las muestras se abren de manera económicamente significativa como categorías de costos dentro de una o más industrias.

\section{Costos pegajosos en empresas de España}

Este trabajo pretende comprobar si en España se observa el comportamiento pegajoso de los costos, en línea con la práctica documentada en otros países. Siguiendo los resultados obtenidos en estudios anteriores, el comportamiento que se espera para las empresas españolas es que cuando los ingresos de explotación se incrementen en un $1 \%$ los gastos de explotación también se incrementen aunque en menor proporción; asimismo, cuando los ingresos de explotación decrezcan los gastos de explotación disminuyan en proporción inferior al incremento que experimentan frente a un aumento de la actividad.

Para validar empíricamente la existencia de costos pegajosos en España, se analizan ingresos y gastos de explotación obtenidos de la base SABI para el periodo 2005-2007 de las empresas que operan en dos sectores muy diferentes, pero emblemáticos para la economía española: fabricación de muebles (CNAE, 2009) y servicios de alojamiento más servicios de restauración (CNAE, 2009). La muestra consta de 194 observaciones de fábricas de muebles y de 1,213 de servicios de alojamiento y restauración. Las variables medidas son las siguientes:

- Ingresos de Explotación (IE) = importe neto de la cifra de ventas + otros ingresos de explotación + ingresos por servicios + otros servicios

- Gastos de Explotación (GE)= consumos de explotación + gastos de personal + dotación para amortización del inmovilizado + variación de provisiones de tráfico y pérdidas de créditos incobrables + otros gastos de explotación

El modelo de Anderson et al. (2003) es utilizado para medir la respuesta o variación de los gastos de explotación frente a cambios en los ingresos de explotación, 
pero discriminando los periodos en que el nivel de actividad e ingresos aumenta o disminuye ${ }^{1}$. Primero se definen los gastos de explotación como función directa de los ingresos de explotación; posteriormente, se determina la relación entre los gastos actuales y los del periodo anterior, haciendo lo mismo para los ingresos de explotación; ambas variables son transformadas logarítmicamente. Para demostrar la existencia o no de costos pegajosos, se introduce una variable dicotómica (Dummy) que toma el valor 1 cuando los ingresos de explotación disminuyen entre los periodos analizados y el valor 0 cuando aumentan o permanecen sin cambio. El modelo de regresión que se utiliza es el siguiente:

$\log \left(\mathrm{GE}_{i, t} / G E_{i, t-1}\right)=\beta_{0}+\beta_{1} \log \left(\mathrm{IE}_{i, t} / I E_{i, t-1}\right)+\beta_{2} *$ Dummy ${ }_{i, t} * \log \left(\mathrm{IE}_{i, t} / I E_{i, t-1}\right)+\varepsilon_{i, t}$

Este modelo permite demostrar empíricamente la existencia de costos pegajosos en empresas españolas. Anderson et al. (2003) señalan que si el modelo tradicional de costos fijos y variables es válido, los cambios (incrementos y disminuciones) en los costos deberían ser iguales por lo que $\beta_{2}=0$. Cuando los ingresos de explotación aumentan, el valor de la variable dicotómica es 0 y el coeficiente $\beta_{1}$ mide el porcentaje de aumento de los gastos frente a un aumento del $1 \%$ en los ingresos de explotación. Por otra parte, cuando los ingresos disminuyen, el valor de la variable dicotómica es 1 y la suma de los coeficientes $\beta_{1}+\beta_{2}$ mide el porcentaje en que disminuyen los gastos de explotación ante una disminución del 1\% en los ingresos de explotación. Si los gastos de explotación son pegajosos, entonces la variación que experimentan ante un aumento en los ingresos de explotación deberá ser mayor que la que experimentan ante disminuciones de dichos ingresos. Por ello, la hipótesis empírica para costos pegajosos es que cuando $\beta_{1}$ es positiva se observará que $\beta_{2}$ es negativa y menor que $\beta_{1}$ en valor absoluto.

\footnotetext{
${ }^{1}$ A pesar de las críticas de Anderson y Lanen (2007) y Balakrishnan et al. (2010), quienes disputan no sólo la relación entre costos pegajosos y decisiones explícitas de los gerentes para cambiar o mantener el nivel de costos, sino también el modelo empírico utilizado para medirlas, entendemos que su uso para las empresas españolas es de utilidad porque permite comparaciones adecuadas.

En un artículo recientemente presentado por Banker et al. (2010), estos autores refutan las críticas de Balakrishnan et al. (2010) y dan cierto y limitado reconocimiento a las de Anderson y Lanen (2007). Indican que las críticas de ambos artículos analizados son algunas preocupaciones acerca de los costos pegajosos, pero que no están actualmente justificadas y que una versión más realista y metodológica de estos estudios soportaría la existencia de costos pegajosos.
} 


\section{Resultados}

Los resultados de las ecuaciones son robustos y concordantes con la hipótesis de que los costos pegajosos también se observan en empresas españolas. La estimación de la ecuación de regresión aplicada a ambos sectores, mueble y alojamiento y restauración, muestra coeficientes significativos al $1 \%$ en todos los casos y al $10 \%$ para $\beta_{2}$ en servicios de alojamiento y restauración:

Fabricación de muebles

$\log \left(\mathrm{GE}_{i, t} / G E_{i, t-1}\right)=-0.002+0.968 \log \left(\mathrm{IE}_{i, t} / I E_{i, t-1}\right)-0.528 * D u m m y * \log \left(\mathrm{IE}_{i, t}\right.$ $\left./ I E_{i, t-1}\right)+\varepsilon_{i, t}$

Servicios de restauración y alojamiento ${ }^{2}$

$\log \left(\mathrm{GE}_{i, t} / G E_{i, t-1}\right)=0.014+0.914 \log \left(\mathrm{IE}_{i, t} / I E_{i, t-1}\right)-0.072 * D u m m y y_{i, t} * \log \left(\mathrm{IE}_{i, t}\right.$ $\left./ I E_{i, t-1}\right)+\varepsilon_{i, t}$

Los valores obtenidos para los sectores del mueble, y restauración y alojamiento, muestran que los gastos de explotación se incrementan en un $0.97 \%$ y $0.91 \%$, respectivamente, por cada $1 \%$ de incremento en los ingresos de explotación; asimismo, los gastos de explotación disminuyen un $0.44 \%$ y $0.84 \%$, respectivamente, por cada $1 \%$ de disminución de los ingresos de explotación.

Se ha calculado una medida denominada "grado de comportamiento pegajoso de los costos" (degree of cost stickiness), según Banker et al. (2010) determinada por $\left(\beta_{1}+\beta_{2}\right) / \beta_{1}$, que arroja los siguientes resultados:

Fabricación de muebles: 0.454

Servicios de restauración y alojamiento: $0.92^{3}$

\footnotetext{
${ }^{2}$ Aplicando el modelo a cada subsector se obtiene lo siguiente: Servicios de restauración (493 observaciones): $\log \left(\mathrm{GE}_{i, t} / G E_{i, t-1}\right)=0.033+0.845 \log \left(\mathrm{IE}_{i, t} / I E_{i, t-1}\right)-0.103 *$ Dummyi,t* $\log \left(\mathrm{IE}_{i, t} / I E_{i, t-1}\right)+\varepsilon_{i, t}$ ( $F=5789.55$, sig. $0.000 ; R^{2}$ ajustado $=0.96 ; V I F=1.13 ; D-W=2.00$ ).

Servicios de alojamiento (720 observaciones):

$\log \left(\mathrm{GE}_{i, t} / G E_{i, t-1}\right)=-0.005+0.994 \log \left(\mathrm{IE}_{i, t} / I E_{i, t-1}\right)-0.110 * D_{u m m y} * \log \left(\mathrm{IE}_{i, t} / I E_{i, t-1-1}+\varepsilon_{i, t}\right.$ $\left(F=1178.73\right.$, sig. $0.000 ; R^{2}$ ajustado $\left.=0.77 ; V I F=1.26 ; D-W=2.07\right)$.

${ }^{3}$ Aplicando el modelo a cada subsector se obtiene lo siguiente:

Servicios de restauración : 0.878

Servicios de alojamiento : 0.889
} 
Basándonos en los fundamentos económicos de los costos pegajosos, puede esperarse que este grado de comportamiento pegajoso varíe sistemáticamente a través de las diferentes industrias y países, con evidencia consistente de esta situación (Banker et al., 2010).

En los dos modelos analizados se confirma la bondad del ajuste realizado y el cumplimento de las hipótesis estadísticas básicas. El modelo aplicado a la fabricación de muebles es altamente significativo de acuerdo con el test de significación global $(F=1832.15, \operatorname{sig}=0.000)$ y el grado de ajuste alcanzado es muy alto $\left(R^{2}\right.$ ajustado=0.95).

El valor del factor de inflación de la varianza $(V I F=1.16)$ indica la ausencia de multicolinealidad elevada entre las variables incluidas en el modelo. El valor del indicador Durbin-Watson $(D-W=2.07)$ muestra la no correlación de las perturbaciones.

La heteroscedasticidad no es un problema importante en los datos; tal como se desprende de los diagramas de dispersión de los residuos, la varianza del error no depende de las variables explicativas del modelo. Por otra parte, el modelo aplicado al sector restauración y alojamiento también es altamente significativo $(F=2963.38$, sig $=0.000)$, y el grado de ajuste es muy alto $\left(R^{2}\right.$ ajustado=0.83). Los valores del factor de inflación de la varianza $(V I F=1.20)$ y del indicador DurbinWatson $(D-W=2.01)$ indican, respectivamente, la ausencia de multicolinealidad entre las variables del modelo y la no correlación de las perturbaciones aleatorias. Los diagramas de dispersión de los residuos muestran que la heteroscedasticidad no es un problema importante en los datos.

\section{Discusión}

Los resultados permiten confirmar que los costos pegajosos se observan en empresas españolas. Sin embargo, se aprecian importantes diferencias entre los dos sectores estudiados, mostrándose el sector Servicios de alojamiento y restauración mucho más flexible a la hora de ajustar sus costos frente a una reducción en el volumen de actividad según se resume en el cuadro 2. La evidencia encontrada apoya el comportamiento diferente de los costos (más o menos pegajosos o incluso no pegajosos) en función de las diferentes industrias estudiadas (Banker et al., 2010). 


\section{Cuadro 2}

Magnitud de costos pegajosos en España

\begin{tabular}{lccc}
\hline Empresas estudiadas & $\begin{array}{c}\text { Efecto de un } \\
\text { aumento del 1\% } \\
\text { en las ventas }\end{array}$ & $\begin{array}{c}\text { Efecto de una } \\
\text { disminución del } \\
\text { 1\% en las ventas }\end{array}$ & $\begin{array}{c}\text { Grado de } \\
\text { comportamiento } \\
\text { pegajoso }\end{array}$ \\
\hline Sector: muebles & Aumentan $0.97 \%$ & Disminuyen $0.44 \%$ & 0.454 \\
\hline $\begin{array}{l}\text { Sector: restauración y } \\
\text { alojamiento }\end{array}$ & Aumentan $0.91 \%$ & Disminuyen $0.84 \%$ & 0.92 \\
\hline Subsector: restauración & Aumentan $0.85 \%$ & Disminuyen $0.74 \%$ & 0.878 \\
\hline Subsector: alojamiento & Aumentan $0.99 \%$ & Disminuyen $0.85 \%$ & 0.889 \\
\hline
\end{tabular}

El primer elemento de los costos que podría ser pertinente para explicar estas diferencias se basa en las relaciones legales que vinculan a las empresas con sus trabajadores. Los contratos a corto plazo parecen ser más habituales en los servicios de alojamiento y restauración que en el sector del mueble. Según datos del Instituto Nacional de Estadística (Europa Press, 2010) para el primer trimestre de 2010, el sector turístico, en general, ha alcanzado una tasa de temporalidad del empleo muy alta $(28.7 \%)$, frente al $30 \%$ del mismo periodo del 2009. Si bien es cierto que este porcentaje varía de manera distinta según los subsectores, el subsector de alojamiento fue uno de los que experimentó un mayor incremento (5\%). Otra tendencia observada es que el aumento de los ocupados se debe principalmente al incremento de los autónomos. En el sector de fabricación del mueble, la situación es opuesta, pues se caracteriza por la gran utilización de contratos indefinidos, donde la mayor parte de los asalariados (58.9\%) tienen entre 25 y 44 años. De hecho, en los últimos años el sector viene experimentando un aumento de los contratos indefinidos y una reducción de los temporales; además, el uso de la jornada a tiempo parcial en este sector es escaso, oscilando solamente entre el $1.2 \%$ y el $3.6 \%$ del total del empleo.

Otra hipótesis que puede explicar la diferencia observada en el grado de costos pegajosos es la localización geográfica de cada tipo de empresa, ya que las fábricas de muebles suelen estar ubicadas en comarcas en las que son la principal fuente de trabajo, lo que supone fuertes presiones sociales para mantener el nivel de gasto a pesar de estar experimentando reducciones en el nivel de actividad. Según datos del Observatorio Industrial del Sector de la Madera y del Mueble (2010), el sector del mueble se caracteriza por su concentración en áreas de especialización productiva claramente definidas; en concreto, con respecto al volumen de negocio generado, ocupan los primeros puestos la comunidad valenciana, con un $17.8 \%$ 
del total de la cifra de negocio del sector en España, Cataluña (15.3\%), Galicia (10.8\%), Andalucía (9.1\%) y Castilla-La Mancha (7.5\%).

Si los factores aquí enunciados permiten explicar la diferencia en el grado de costos pegajosos observados en estos sectores tradicionales de la economía española, entonces los resultados obtenidos en este estudio deberían servir para llamar la atención a los gerentes sobre cómo manejar los costos pegajosos. Los gerentes pueden evaluar su exposición a los costos pegajosos analizando la sensibilidad de los cambios en los costos ante una reducción del volumen de actividad. Pueden tomarse ciertos cuidados para hacer los costos más sensibles ante una caída en la actividad; por ejemplo, mediante contratos de más corto plazo, tercerizaciones, etc., tal como se sugiere en el cuadro 3.

\section{Cuadro 3 \\ Reconocer y controlar los costos pegajosos}

\begin{tabular}{|c|c|c|}
\hline $\begin{array}{l}\text { Tipo de } \\
\text { costo }\end{array}$ & $\begin{array}{l}\text { Reconocer costos } \\
\text { pegajosos }\end{array}$ & Controlar costos pegajosos \\
\hline $\begin{array}{l}\text { Posiciones no } \\
\text { críticas }\end{array}$ & $\begin{array}{l}\text { Contratos indefinidos } \\
\text { y fuerte presencia sindical }\end{array}$ & $\begin{array}{c}\text { Contratos por tiempo limitado con renovaciones } \\
\text { sujetas a renegociación. } \\
\text { Ejemplos: operarios de línea de montajes } \\
\text { y camareros }\end{array}$ \\
\hline $\begin{array}{c}\text { Costos } \\
\text { operativos }\end{array}$ & $\begin{array}{c}\text { Compromisos } \\
\text { significativos e ilimitados } \\
\text { en el tiempo }\end{array}$ & $\begin{array}{l}\text { Proyectos de duración limitada y recursos } \\
\text { identificables. } \\
\text { Ejemplos: tercerizar logística de distribución } \\
\text { y aprovisionamiento }\end{array}$ \\
\hline $\begin{array}{l}\text { Costos no } \\
\text { operativos }\end{array}$ & $\begin{array}{c}\text { Compromisos } \\
\text { significativos e ilimitados } \\
\text { en el tiempo }\end{array}$ & $\begin{array}{c}\text { Proyectos de duración limitada y recursos } \\
\text { identificables. } \\
\text { Ejemplos: tercerizar servicio de limpieza de la } \\
\text { planta/taller, lavandería, troceado de verduras, etc. }\end{array}$ \\
\hline
\end{tabular}

\section{Conclusión}

Comprender el comportamiento de los costos es fundamental para administrar cualquier organización, pero la teoría aprendida de que hay costos variables no es un fiel reflejo de la práctica. La evidencia, en general, muestra que en el corto y mediano plazos los costos son pegajosos; esto es, aumentan más ante un incremento de la actividad que lo que decrecen cuando el volumen de actividad baja. Una explicación de este comportamiento es la demora de los gerentes para reducir 
costos cuando disminuye el volumen de actividad, en una suerte de fuerzas contrapuestas entre mantener el costo de la capacidad ociosa e incurrir el costo de salida y reemplazo de los recursos desechados.

Por ello, es fundamental salirse del modelo tradicional de costos (Hongren, 2002) que indica que un costo variable cambia proporcionalmente ante variaciones en el factor o generador de costos, cualquiera que sea la dirección del mismo.

Es importante tener en cuenta que la rigidez de los costos a la baja no se genera por su comportamiento intrínseco, sino que es producto de las decisiones de los gerentes. Los costos pegajosos pueden reconocerse analizando la sensibilidad de las variaciones de los costos ante reducciones en el volumen de actividad; además, pueden ser controlados haciéndolos más sensibles ante una caída en la actividad, por ejemplo, mediante contratos de más corto plazo, tercerizaciones, etcétera.

Los resultados obtenidos en este estudio muestran que el concepto de costos pegajosos se observa en empresas españolas en el periodo 2005-2007. La relación entre el aumento de los ingresos y el aumento de los gastos es positiva; por tanto, ante una expansión de la actividad los costos crecerán, pero menos que proporcionalmente; por otra parte, si la actividad se contrae en periodos posteriores, se observará que los gastos son inflexibles a la baja, por lo que los costos se reducirán, pero no en la misma proporción que lo hicieron cuando el volumen de actividad creció. En los sectores estudiados, muebles, alojamiento y restauración, un aumento del $1 \%$ en los ingresos de explotación provoca un incremento de los gastos de explotación en un $0.97 \%$ y $0.91 \%$, respectivamente; sin embargo, cuando los ingresos de explotación caen en un $1 \%$ dichos gastos se reducen sólo en $0.44 \%$ y $0.84 \%$. Uno de los factores que puede contribuir a explicar las diferencias entre dichos sectores es la utilización de contratos laborales indefinidos en el sector del mueble frente a la importante proporción de contratos temporales en la industria turística; asimismo, podría contribuir a dicha explicación la concentración de fábricas de muebles en ciertas comarcas, lo que las lleva a ser un actor importante en la economía local, con las consiguientes presiones sociales, gubernamentales y sindicales por la no-reducción de empleos y la petición de suministro de bienes y servicios provistos localmente.

Similares resultados podrían encontrarse en empresas de diversos sectores en otros contextos internacionales. La idea que subyace al comportamiento de costos pegajosos es muy poderosa, pero para ser aceptada necesita contar con múltiples 
contrastaciones empíricas en diversos entornos macroeconómicos, así como en diversas industrias (Balakrishnan et al., 2010). Este trabajo muestra que la metodología capaz de identificar el citado comportamiento de los costos puede ser potencialmente aplicable a otros contextos, siempre que se disponga de una base de datos de ingresos y gastos organizados por industria. El desafío para los investigadores locales, que se presenta como una interesante oportunidad de investigación, es obtener los datos de empresas del medio para replicar el modelo y así respaldar o contradecir la idea generalmente aceptada de que los costos variables tienen un comportamiento pegajoso a pesar de la teoría aprendida y enseñada constantemente en los claustros (Hongren, 2002).

\section{Referencias}

Anderson, M.C., R.D. Banker y S.N. Janakiraman (2003). Are selling, general and administrative costs "sticky"? Journal of Accounting Research 41 (1): 47-63.

Anderson, S. y W. Lanen (2007). Understanding cost management: what can we learn from the empirical evidence on "sticky costs"? Disponible en: http:// ssrn.com/abstracts $=975135$

Balakrishnan, R., M.J. Petersen y N. Soderstrom (2004). Does capacity utilization affect the "stickiness" of cost? Journal of Accounting, Auditing \& Finance 19 (3): 283-299.

y T.S. Gruca (2008). Cost stickiness and core competence: a note. Contemporary Accounting Research 25 (4): 993-1006.

E. Labro y N. Soderstrom (2010). Cost structure and sticky costs. Disponible en: http://ssrn.com/abstracts=1562726

Banker, R. D., M. Ciftci y R. Mashruwala (2008). Managerial optimism, prior period sales changes and sticky cost behaviour Disponible en: http://papers.ssrn.com/so13/papers.cfm?abstract_id=902546.

Banker, R., D. Byzalov y J. Plehn - Dujowich (2010). Sticky cost behaviour: theory and evidence. AAA 2011 Management Accounting Section (MAS) Meeting paper. Disponible en: http://ssrn.com/abstract=1659493 
Chen, C., H. Lu y T. Sougiannis (2007) Managerial empire building, corporate governance, and the asymmetrical behaviour of selling, general, and administrative costs. Disponible en: http://papers.ssrn.com/so13/papers. cfm?abstract_id=1014088

Calleja, K., M. Steliaros y D.C. Thomas (2006). A note on cost stickiness: some international comparisons. Management Accounting Research (17):127-140.

CNAE (2009), Lista completa de actividades. Disponible en: http://www.cnae. com.es/lista-actividades.php

EUROPA PRESS (2010) Madrid 07/05/2010. Disponible en: http://www.europapress.es/economía

Hongren, C., G. Foster y S. Datar (2002). Contabilidad de costos, un enfoque gerencial. $10{ }^{\circ}$ ed. México: Pearson,

Mak, Y. T. y M.L. Rousch (1994). Flexible budgeting and variance analysis in an activity - based cost environment. Accounting Horizons, June 8 (2): 93-103.

Malcom, R.E. (1991). Overhead control implications of activity costing. Accounting Horizons: 69-78.

Noreen, E. y N. Soderstrom (1997). The accuracy of proportional cost models: evidence from hospital service departments. Review of Accounting Studies (2): 89-114.

Observatorio industrial del sector de la madera y del mueble (2010): Estrategia de modernización del sector de la madera y del mueble, Confemadera y Observatorio industrial de la madera. Disponible en: http://www.observatorioindustrialdelamadera.com/informes_publicaciones/Conclusiones_recomendaciones_OIMadera_definitivas.pdf

Porporato, M. y E. Werbin (2010). Active cost management in banks: evidence of sticky costs in Argentina, Brazil and Canada. AAA 2011 Management Accounting Section (MAS) Meeting Paper. Disponible en: http://ssrn.com/ abstract $=1659228$ 
Ribeiro de Medeiros, O. y P. de Souza Costa (2004). Cost stickiness in Brazilian firms. Disponible en: http://papers.ssrn.com/so13/papers.cfm?abstract_ $\mathrm{id}=632365$

Weiss, D. (2010). Cost behaviour and analyst's earnings forecasts. The Accounting Review 85 (4): 1441.

Werbin, E. (2009) Costos pegadizos (sticky costs). Revista Iberoamericana de Contabilidad de Gestión 7 (14), Artículo 2. Disponible en: www.observatoriolatinoamericano.com/

West, D. A. (2003). Three financial strategies. Journal of Health Care Finance 30 (1): 10-22.

Wiersma, E (2010). The impact of the reward structure on stickiness. AAA 2011 Management Accounting Section (MAS) Meeting Paper. Disponible en: http://ssrn.com/abstract=1668758 